\title{
Calculating Newton's Gravity (Big G) from Coulomb, Lorentz and Centripetal Force
}

\author{
Greg Poole \\ Industrial Tests, Inc., Rocklin, CA, USA \\ Email: greg@indtest.com
}

How to cite this paper: Poole, G. (2019) Calculating Newton's Gravity (Big G) from Coulomb, Lorentz and Centripetal Force. Journal of High Energy Physics, Gravitation and Cosmology, 5, 623-628. https://doi.org/10.4236/jhepgc.2019.53034

Received: April 26, 2019

Accepted: May 18, 2019

Published: May 21, 2019

Copyright $\odot 2019$ by author(s) and Scientific Research Publishing Inc. This work is licensed under the Creative Commons Attribution International License (CC BY 4.0).

http://creativecommons.org/licenses/by/4.0/

\begin{abstract}
The velocity of the Earth around the Sun and its corresponding mass are used in a standard centripetal force equation to match what Isaac Newton calculated with the Universal Law of Gravitation in 1687. Electromagnetic calculations are used to prove that electromagnetic forces are an insignificant contributor to the centripetal force or gravity, but do provide the bending force for the planets to maintain a circular orbit around the Sun. It is thus proven using classical physics that the force of gravity is a simple centripetal force with a very small electromagnetic force contribution.
\end{abstract}

\section{Keywords}

Gravity, Big G, Coulomb Law, Lorentz Law, Centripetal Force

\section{Introduction}

In 1687 Isaac Newton published Philosophiae Naturalis Pincipia Mathematica [1]-Newton's pronounced three laws of motion and a law of universal gravitation. The manuscript was a revolutionary set of principles which applied not only to the Earth but also to the heavens. Their simplicity and extremely universal applicability forever changed physics and astronomy. While Newton's universal law of gravitation does not explain what gravity is, it does say how the force of gravity works. The purpose of this paper is to complete Newton's work and mathematically prove with the same simplicity what gravity is and how it occurs between suns and planets.

\section{Force of Gravity between Sun and Earth}

Newton's Law of Universal Gravitation determined that every point mass attracts every other point mass in the universe by a force. This force is proportion- 
al to the masses of the objects and inversely proportional to their separation. This attractive force always points the two objects inward (Figure 1). Two large bodies, like planets, can be considered as point-like masses, as the distance between them is very large compared to their sizes. For our paper the mass of each planet or sun can be represented as a point mass located at its center-of-mass.

The force of gravity between two objects is given by the formula

$$
F=\frac{G m_{1} m_{2}}{r^{2}}
$$

where $G \approx 6.674 \times 10^{-11} \mathrm{Nm}^{2} / \mathrm{kg}^{2}$ is the gravitational constant.

For the Sun-Earth system we have:

Mass of the Sun, $m \approx 2 \times 10^{30} \mathrm{~kg}$,

Mass of the Earth, $m \approx 6 \times 10^{24} \mathrm{~kg}$,

Separation, $r \approx 1.5 \times 10^{11}$ meters.

Therefore, the gravitational force between the Sun and Earth is approximately

$$
F \approx 6.7 \times 10^{-11} \times 12 \times 10^{54} \div 2.25 \times 10^{22} \approx 3.6 \times 10^{22} \mathrm{~N} .
$$

\section{Coulombs Law Applied to Sun and Earth}

Representing the Sun and Earth as distant charged particles we can approximate the electrostatic force between the planets. Coulomb's law [2], otherwise known as Coulomb's inverse-square law, describes the electrostatic force acting between two charges. The force acts along the shortest line that joins the charges. It is repulsive if both charges have the same sign, and attractive if they have opposite signs (Figure 2).

Coulomb's law is formulated as follows:

$$
F=\frac{K_{e} q_{1} q_{2}}{r^{2}}
$$

where:

$F$ is the electrostatic force between charges (in Newtons),

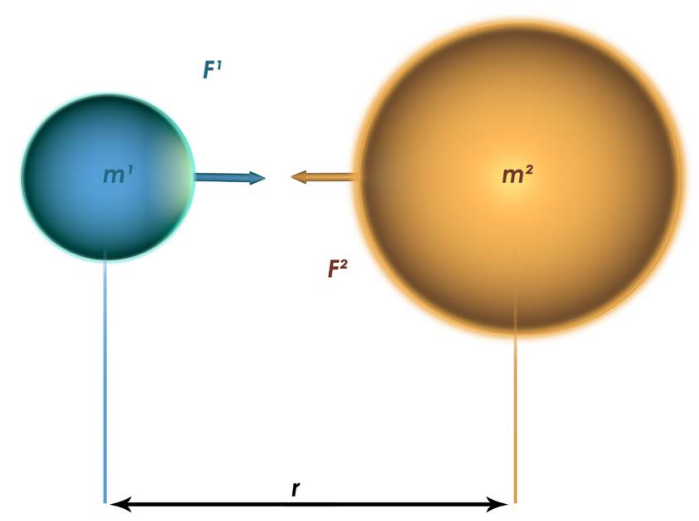

Figure 1. The force between two objects is proportional to the masses of the objects and inversely proportional to their separation. This attractive force always points the two objects inward. 


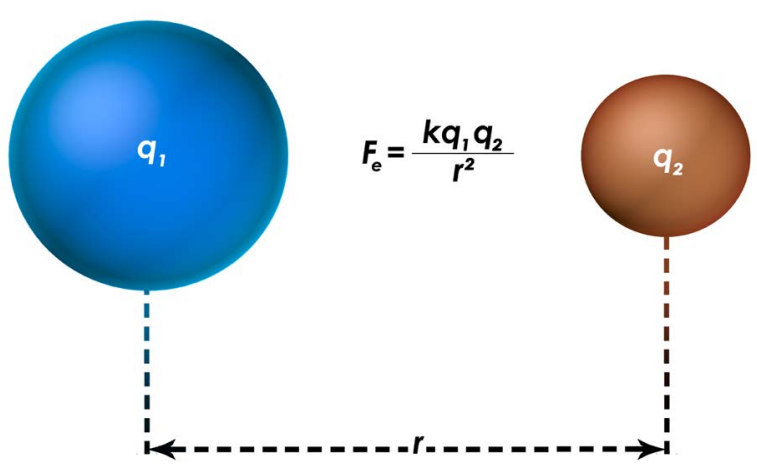

Figure 2. The force acts along the shortest line that joins the charges. It is repulsive if both charges have the same sign, and attractive if they have opposite signs.

$q_{1}$ is the magnitude of the first charge (in Coulombs), for the Earth this value has been well described and is calculated to be $4 \times 10^{5} \mathrm{C}$, based on a $100 \mathrm{~V} / \mathrm{m}$ near the surface of the Earth.

$q_{2}$ is the magnitude of the second charge (in Coulombs), for the Sun this value is calculated to be $2.6 \times 10^{12} \mathrm{C}$, based on a surface radiation of the Sun at $6.33 \times$ $10^{7} \mathrm{~W} / \mathrm{m}^{2}$ [3], which equates to $154,480 \mathrm{~V} / \mathrm{m}$ near the surface of the Sun. This value of electrical field intensity is readily derived from Watts $=V^{2} / R$, where $R$ is the value of free space or air, which is equal to $377 \mathrm{ohms}$.

$r$ is the shortest distance between the charges (in $\mathrm{m}$ ), 150 million kilometers is the distance from the Earth to the Sun.

$K_{e}$ is Coulomb's constant, $9.0 \times 10^{9} \mathrm{Nm}^{2} / \mathrm{C}^{2}$.

Using Coulombs Law, we can calculate the electrostatic force between the Sun and Earth to be, $\mathrm{F}=415,425$ Newtons.

\section{Lorentz Law Applied to the Sun and Earth}

The Lorentz Force Law [4] states that a magnetic field exerts a force on a moving charged particle, or in our case a planet. The value of the force depends on the charge, velocity and strength of the magnetic field. The direction of the Lorentz force is perpendicular to both the particle direction and to the magnetic field. As an orbital effect, the Earth's trajectory bends in the Sun's massive magnetic field (Figure 3).

$$
F=q v B \sin (\alpha)
$$

where

$q$ is the particle's charge; for the Earth we use 400,000 Coulombs $v$ is the particle's velocity; for the Earth the value is $30,000 \mathrm{~m} / \mathrm{s}$

$B$ is the strength of the magnetic field; for the Earth the value is 0.00005 Tesla $\alpha$ is the angle between the direction of the particle's trajectory and the direction of the magnetic field; for simplicity we set the angle to 90 degrees.

$F$ is the resulting force.

Using Lorentz Law, we can thus calculate the magnetic force between the Sun and Earth to be, $F=600,000$ Newtons. 


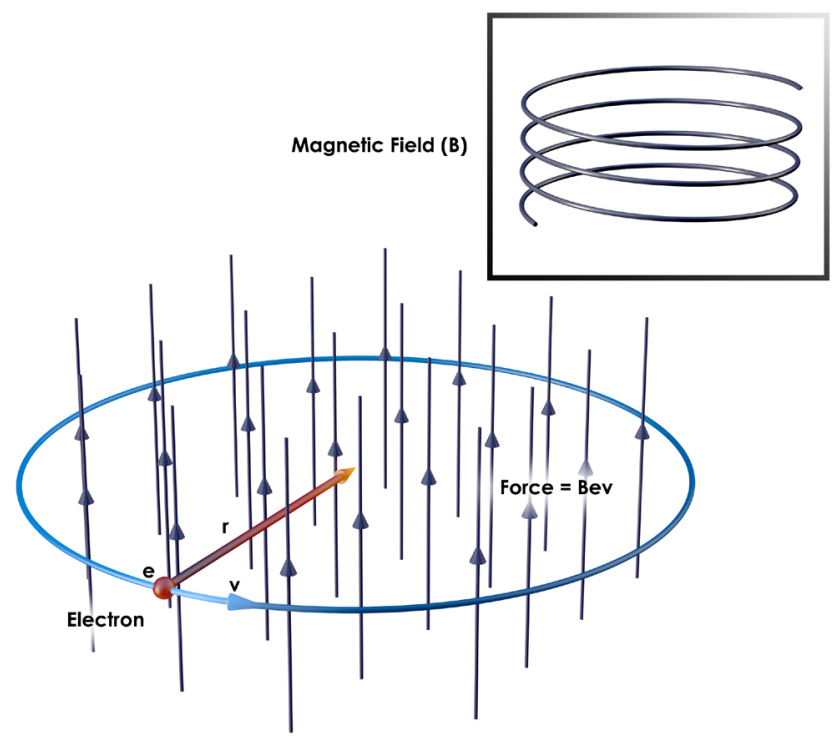

Figure 3. The direction of the Lorentz force is perpendicular to both the particle direction and to the magnetic field. As an orbital effect, the Earth's trajectory bends in the Sun's massive magnetic field.

The combined electromagnetic force can be calculated by adding Coulomb's Force with Lorentz Force. The total electromagnetic force is slightly over $1,000,000$ Newtons. Thus, we have shown that the electromagnetic forces combined are nowhere near Newton's calculated force of gravity or big G for the Earth and Sun.

\section{Centripetal Force Applied to the Sun and Earth}

Any motion in a curved path represents accelerated motion, and requires a force directed toward the center of the curvature of the path (Figure 4). This force is called the centripetal force which means "center seeking" force [5]. The equation for centripetal force is described as follows:

$$
F_{\text {centripetal }}=m \frac{v^{2}}{r}
$$

where,

- $m=$ mass; the mass of the Earth is $5.972 \times 10^{24} \mathrm{~kg}$.

- $V=$ velocity; for the Earth this is $30,000 \mathrm{~m} / \mathrm{s}$ around the Sun.

- $r$ = radius or distance from Earth to Sun 150 million kilometers

Inserting the values, we obtain a Centripetal Force of $3.54 \times 10^{22}$ Newtons. This value is within $0.005 \%$ of Newton's calculated value. The calculations for the other planets are shown in Table 1.

\section{Conclusion}

My calculations demonstrate that the Centripetal force caused simply by the Earth rotating around the Sun to be the primary source of gravity or Big G between planets. This work complements my first paper which theorized that little 


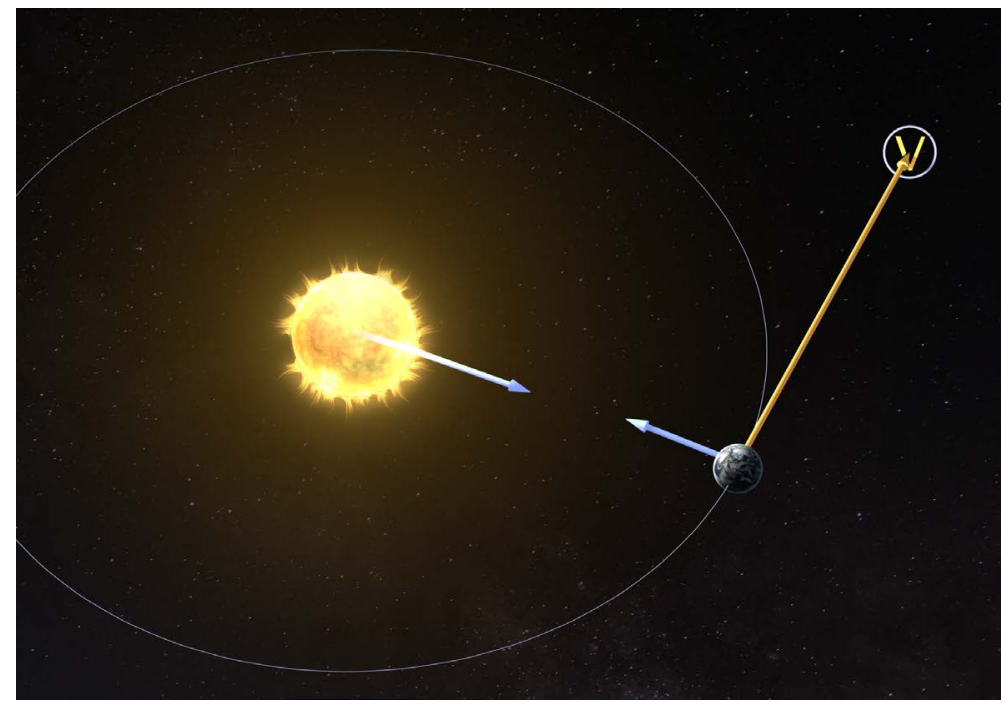

Figure 4. Any motion in a curved path represents accelerated motion, and requires a force directed toward the center of the curvature of the path.

Table 1. Calculations for centripetal force and comparison to Newton's calculated value.

\begin{tabular}{ccccccc}
\hline Planet & $\begin{array}{c}\text { Newton's Law } \\
\text { Force }(\mathrm{N})\end{array}$ & $\begin{array}{c}\text { Mass } \\
(\mathrm{Kg})\end{array}$ & $\begin{array}{c}\text { Velocity } \\
(\mathrm{m} / \mathrm{s})\end{array}$ & $\begin{array}{c}\text { Distance } \\
(\mathrm{m})\end{array}$ & $\begin{array}{c}\text { Centripetal } \\
\text { Force }(\mathrm{N})\end{array}$ & $\begin{array}{c}\text { Error } \\
(\%)\end{array}$ \\
\hline Mercury & $1.31 \mathrm{E}+22$ & $3.30 \mathrm{E}+23$ & 47400 & $5.79 \mathrm{E}+10$ & $1.28 \mathrm{E}+22$ & $2.3 \%$ \\
Venus & $5.54 \mathrm{E}+22$ & $4.87 \mathrm{E}+24$ & 35000 & $1.08 \mathrm{E}+11$ & $5.52 \mathrm{E}+22$ & $0.4 \%$ \\
Earth & $3.54 \mathrm{E}+22$ & $5.98 \mathrm{E}+24$ & 29800 & $1.50 \mathrm{E}+11$ & $3.54 \mathrm{E}+22$ & $0 \%$ \\
Mars & $1.64 \mathrm{E}+21$ & $6.42 \mathrm{E}+23$ & 24000 & $2.28 \mathrm{E}+11$ & $1.62 \mathrm{E}+21$ & $1.2 \%$ \\
Jupiter & $4.16 \mathrm{E}+23$ & $1.90 \mathrm{E}+27$ & 13000 & $7.78 \mathrm{E}+11$ & $4.12 \mathrm{E}+23$ & $1 \%$ \\
Saturn & $3.69 \mathrm{E}+22$ & $5.68 \mathrm{E}+26$ & 9700 & $1.43 \mathrm{E}+12$ & $3.73 \mathrm{E}+22$ & $1.08 \%$ \\
Uranus & $1.40 \mathrm{E}+21$ & $8.68 \mathrm{E}+25$ & 6800 & $2.87 \mathrm{E}+12$ & $1.40 \mathrm{E}+21$ & $0 \%$ \\
Neptune & $6.68 \mathrm{E}+20$ & $1.02 \mathrm{E}+26$ & 5400 & $4.50 \mathrm{E}+12$ & $6.62 \mathrm{E}+20$ & $0.9 \%$ \\
Pluto & $5.55 \mathrm{E}+16$ & $1.46 \mathrm{E}+22$ & 4700 & $5.91 \mathrm{E}+12$ & $5.46 \mathrm{E}+16$ & $1.6 \%$ \\
\hline
\end{tabular}

$\mathrm{g}$ is also a centripetal force [6]. The electrostatic and magnetic forces are considered negligible contributors to the centripetal force, though it is postulated that the magnetic force is what sets the Earth into orbit and guides its bending pattern around the Sun. The Sun's magnetic field is many times greater than Earth's magnetic field. The Sun is nearly 12,000 times the size of the Earth and my calculations show the electric field to be 1500 times. The Sun's magnetic field has seen spikes of 0.3 Tesla or 3000 Gauss, [7] which is many times that of the Earth's magnetic field. The planets' elliptical pattern around the Sun is caused by planets passing through the Sun's electromagnetic field. Planets behave similar to particles passing through an electromagnetic field in this regard. The speed at which the planets travel, and the mass of the planets, determines the centripetal or gravitational force between the planets and the Sun. The true nature of gravity as a combined centripetal and electromagnetic force is thus explained. 


\section{Acknowledgements}

The author wishes to acknowledge ASK Scientific (https://www.askscientific.com) for the formatting assistance. I am also grateful to high school physics teacher Sam Kern who first proposed to his students a rotating body around the earth as a source of gravitation.

\section{Conflicts of Interest}

The author declares no conflicts of interest regarding the publication of this paper.

\section{References}

[1] Newton, I. (2010) The Principia: Mathematical Principles of Natural Philosophy. Snowball Pub, 10.

[2] Huray, P.G. (2010) Maxwell's Equations. Wiley, Hoboken. https://doi.org/10.1002/9780470549919

[3] Bhatia, S.C. (2014) Advanced Renewable Energy Systems, (Part 1 and 2). WPI Publishing, New Delhi. https://doi.org/10.1201/b18242

[4] Jackson, J.D. (1999) Classical Electrodynamics. 3rd Edition. Wiley, New York.

[5] Tipler, P.A. and Mosca, G. (2003) Physics for Scientists and Engineers. 5th Edition. Macmillan, 129.

[6] Poole, G. (2017) Theory of Electromagnetism and Gravity-Modeling Earth as a Rotating Solenoid Coil. Journal of High Energy Physics, Gravitation and Cosmology, 3, 663-692. https://doi.org/10.4236/jhepgc.2017.34051

[7] NASA Cosmos. https://ase.tufts.edu/cosmos/view_chapter.asp?id=26\&page=2 